\title{
The association between neurocognitive functioning and clinical features of borderline personality disorder
}

\author{
Bahar Kaplan, ${ }^{1}$ Medine Yazici Gulec, ${ }^{2}$ Sakir Gica, ${ }^{3}$ iD Huseyin Gulec ${ }^{2}$ \\ ${ }^{1}$ Department of Psychiatry, Sivas Numune Hospital, Sivas Provincial Health Directorate, Sivas, Turkey. ${ }^{2}$ Department of Psychiatry, University \\ of Health Sciences Turkey, Erenkoy Mental Health and Neurological Disease Education and Research Hospital, Istanbul, Turkey. ${ }^{3}$ Department \\ of Psychiatry, Meram Medical Faculty, Necmettin Erbakan University, Konya, Turkey.
}

\begin{abstract}
Objective: To investigate the relationship between neurocognitive profiles and clinical manifestations of borderline personality disorder (BPD).

Methods: Forty-five patients diagnosed with BPD and 35 healthy volunteers were included in the study. The BPD group was evaluated with the Borderline Personality Inventory for dissociative, impulsivity and suicidal dimensions. The Verbal Memory Processes Test and the Cambridge Neurophysiological Assessment Battery were administered to both the BPD and healthy control groups.

Results: BPD patients differed from controls in sustained attention, facial emotion recognition, and deteriorated verbal memory function. A model consisting of the Dissociative Experiences Scale Taxon (DES-T), motor impulsivity and Scale for Suicidal Behavior scores explained $52 \%$ of the variance in Borderline Personality Inventory scores. It was detected that motor impulsivity, decisionmaking and recognizing sadness may significantly predict DES-T scores, and response inhibition and facial emotion recognition scores may significantly predict impulsivity.

Conclusion: Our findings demonstrate that the disassociation, impulsivity, and suicidality dimensions are sufficient to represent the clinical manifestations of BPD, that they are related to neurocognitive differences, and that they interact with clinical features.
\end{abstract}

Keywords: Emotion recognition; suicidality; dissociation; impulsivity; decision-making

\section{Introduction}

Borderline personality disorder (BPD) is characterized by a troublesome pattern of instability in affect regulation, self-image, impulse control, and interpersonal relationships, which leads to emotional dysregulation, impulsive aggression, repetitive self-harm/suicidal tendencies, and clinical symptoms. ${ }^{1,2}$ BPD is a complex multidimensional disorder with a wide range of symptoms and cognitive deficits that is frequently accompanied by other psychiatric disorders. ${ }^{3}$ BPD diagnosis is impeded by overlapping disorders and lack of a biological profile or specific family history. ${ }^{4}$ To ameliorate the diagnosis problem, the DSM-5 offers a hybrid evaluation model that includes a dimensional approach. Dissociative symptoms (1001, 1002), impulsivity, and suicidality (1003) are seen as common dimensions in BPD and play an important role in clinical presentation, according to recent studies investigating the dimensional aspect (DSM-5) of the disorder, whose importance is now receiving more attention. Borderline personality disorder has been shown to be a result of neurobiological detriment, primarily executive function, due to complicated neuronal connections, ${ }^{5}$ mainly in the

Correspondence: Sakir Gica, Necmettin Erbakan University, Meram Medical Faculty, Yunus Emre Mah. Beyşehir Cad. No: 281, Konya, Turkey.

E-mail: sakirgica@ hotmail.com

Submitted Oct 15 2019, accepted Jan 14 2020, Epub Apr 172020. prefrontal cortex and other brain regions, ${ }^{6,7}$ and that changes in executive function can be seen as an endophenotypic trait. ${ }^{8}$ Although the clinical features of BPD are thought to be the result of neurodevelopmental neurocognitive functions, they are not considered a neurocognitive disorder. In addition, impaired neurocognitive function is associated with clinical functioning, ${ }^{8,9}$ general functioning ${ }^{10}$ and continuation of treatment. ${ }^{8}$ Social cognition, a prominent neurocognitive function, is involved in the frequent interpersonal problems and affective dysregulation in BPD. ${ }^{11}$ In interpersonal relationships, the correct interpretation of emotions (e.g., the accurate recognition of facial emotions) is an important cognitive component of intact social functioning. ${ }^{12}$ Disturbed emotion recognition has been shown in BPD.

The aim of the present study was to further investigate the relationship between neurocognitive profiles and clinical manifestations by correlating the Verbal Memory Processes Test (VMPT) and a computerized version of the Cambridge Neurophysiological Assessment Battery (CANTAB). We think that the dissociation, impulsivity and suicidality dimensions should be discussed as representative of BPD and that they may contribute to the problem of ongoing
How to cite this article: Kaplan B, Yazici Gulec M, Gica S, Gulec H. The association between neurocognitive functioning and clinical features of borderline personality disorder. Braz J Psychiatry. 2020;42:502-509. http://dx.doi.org/10.1590/1516-4446-2019-0752 
diagnostic validity in this disorder. As a preliminary stage of the investigation, the diagnostic validity of these dimensions will be tested by considering whether a model including dissociation, impulsivity and suicidal behaviors can sufficiently represent the disorder. Adding cognitive deficits to the most common aspects of the clinical presentation could provide a different approach to assessing BPD. Our hypotheses were that the BPD group would have a different neurocognitive profile than healthy controls and that this profile would be related to the impulsivity, suicidal behavior, dissociation dimensions.

\section{Methods}

\section{Participants}

This was a case-control study with a prospective nature. The sample included 45 patients who were admitted to the University of Health Sciences Turkey, Erenkoy Mental Health and Neurological Disease Education and Research Hospital between September 2015 and May 2016 and were diagnosed as having BPD according to DSM-5 criteria. Each patient was informed about the study and agreed to participate. The control group consisted of 35 healthy individuals who were matched with the BPD group on the basis of age, sex, and education level. The Structured Clinical Interview for DSM-IV Axis I Disorders (SCID-I) and the Structured Clinical Interview for DSM-III-R Personality Disorders (SCID-II) were applied to all participants. Patients under 18 or over 65 years of age, those with a lack of education that might hinder compliance with the instructions, those with a comorbid psychiatric disorder, those who had noticeable mental retardation or cognitive decline in the psychiatric examination, those who were diagnosed through the interview as having a serious general medical condition, and those who were diagnosed with alcohol/substance use disorder were excluded from the study.

\section{Procedure}

BPD patients were assessed with the Borderline Personality Inventory (BPI) dissociative, impulsivity and suicidal behavior dimensions, as well as for other comorbid psychiatric conditions. The VMPT and CANTAB were administered to both the BPD and control groups; no other clinical evaluation scales were used. The neurocognitive tests were applied in a silent room. Brief instructions were provided before each test battery. The individuals were accompanied by a researcher, who also administered the tests. The tests took approximately 1 hour 45 minutes to complete.

\section{Data collection tools}

Data was collected through the following instruments: a sociodemographic data collection form, the BPI, the SCID-I and SCID-II, the Dissociative Experiences Scale Taxon (DES-T), the Barratt Impulsivity Scale, the Scale for Suicidal Behavior (SSB), the VMPT, the CANTAB, the Cambridge Gambling Task (CGT- to assess decision implementation skills), a paired-associate learning test (to assess visual memory and learning), the Stop-Signal Task (to assess the motor response inhibition), a Rapid Visual Processing task (to assess sustained attention), the Intra-Extra Dimensional Set Shifting Test (to assess rule learning, reverse learning and attention set shifting), and the Emotional Recognition Task (ERT - to assess emotion recognition through facial expressions).

\section{Statistical analysis of the data}

In SPSS version 16.0, descriptive statistical methods (mean, standard deviation, frequency, percentage) were used to analyze the study data. The compatibility of nonnormal distributions were visually (histogram and probability graphics) and analytically (Kolmogorov-Smirnov and Shapiro-Wilk tests) examined. An independent Student's $t$-test was also used to compare cognitive, sociodemographic, parametric and quantitative data between groups, while the chi-square test was used to compare categorical data. Multivariate linear regression analysis was used to evaluate the effect of clinical features, such as dissociative findings, suicidality and impulsivity in BPI scores. Separate multivariate linear stepwise regression models were created to investigate the effects of cognitive data on clinical features. The data are presented as means and $95 \%$ confidence intervals, with a significance level of $p<0.05$.

According to calculations performed in $\mathrm{G}^{*}$ Power 3.1.9.2 (effect size: 0.8 , significant tail: 2), the study power was $0.93 .^{13,14}$

\section{Ethics statement}

This study was approved by the clinical research ethics committee of the Erenkoy Mental Health and Neurological Disease Education and Research Hospital.

\section{Results}

The study included 45 patients, aged between 18 and 47 years, who were being followed up after BPD diagnosis. Thirty-five healthy individuals aged $20-36$ years were recruited as the control group. The sociodemographic information and the comparisons between the groups are presented in Table 1.

In the BPD group, 33 patients had attempted suicide and 21 had a history of hospitalization.

The neurocognitive test scores of the BPD and control groups were compared, and the results are shown in Table 2.

A model was developed including impulsivity, suicidal ideation, and dissociative symptoms, which cause great problems, are difficult to manage, and require substantial follow-up in the clinical course of BPD. Suicidal ideation scale scores, total Barratt Impulsivity Scale scores and sub-scores (inability to plan, motor impulsivity and impulsivity in attention), and DES-T scores were approached as independent variables, and their representation in BPI scores was examined in Table 3 using stepwise multivariate regression analysis. According to the results, 
Table 1 Comparison of sociodemographic characteristics in the borderline personality disorder and control groups

\begin{tabular}{|c|c|c|c|c|}
\hline & BPD $(n=45)$ & Control $(n=35)$ & $t^{*} / \chi^{2}$ & $p$-value \\
\hline $\begin{array}{l}\text { Gender } \\
\text { Female } \\
\text { Male }\end{array}$ & $\begin{array}{c}42(93.3) \\
3(6.7)\end{array}$ & $\begin{array}{c}30(85.7) \\
5(14.3)\end{array}$ & 1.270 & 0.260 \\
\hline Age, mean $\pm S D$ & $27.3 \pm 6.3$ & $27.5 \pm 4.3$ & 2.798 & 0.848 \\
\hline Education (years), mean \pm SD & $11.7 \pm 2.6$ & $12.7 \pm 2.5$ & 0.225 & 0.089 \\
\hline $\begin{array}{l}\text { Marital status } \\
\text { Single } \\
\text { Married } \\
\text { Divorced }\end{array}$ & $\begin{array}{c}33(73.3) \\
7(15.6) \\
5(11.1)\end{array}$ & $\begin{array}{c}23(65.7) \\
12(34.3) \\
-\end{array}$ & 6.960 & $0.031^{*}$ \\
\hline $\begin{array}{l}\text { Occupation } \\
\text { Employee } \\
\text { Unemployed } \\
\text { Student }\end{array}$ & $\begin{array}{c}19(42.2) \\
19(42.2) \\
7(15.6)\end{array}$ & $\begin{array}{c}29(82.9) \\
- \\
6(17.1)\end{array}$ & 20.226 & $<0.001^{*}$ \\
\hline
\end{tabular}

Data presented as $\mathrm{n}(\%)$, unless otherwise specified.

$\mathrm{BPD}=$ borderline personality disorder; $\mathrm{SD}=$ standard deviation.

$* p<0.05$; chi-square and Student's $t$-test were performed.

\begin{tabular}{|c|c|c|c|c|}
\hline Test/Outcome parameter & BPD $(n=45)$ & Control $(n=35)$ & $t / \chi^{2}$ & $p$-value \\
\hline \multicolumn{5}{|l|}{ PAL } \\
\hline Total errors (adjusted) & $25.1 \pm 24.7$ & $18.2 \pm 16.3$ & 1.566 & 0.160 \\
\hline \multicolumn{5}{|l|}{ RVP } \\
\hline Ability to determine target directory & $0.86 \pm 0.5$ & $0.89 \pm 0.5$ & 0.379 & $0.041 *$ \\
\hline \multicolumn{5}{|l|}{ IED } \\
\hline Total errors (adjusted) & $26.2 \pm 20.9$ & $20.2 \pm 18.6$ & 2.463 & 0.190 \\
\hline \multicolumn{5}{|l|}{ VMPT } \\
\hline Immediate memory & $6.1 \pm 1.7$ & $6.7 \pm 1.5$ & 0.126 & 0.100 \\
\hline Complete learning points & $115.6 \pm 15.4$ & $129.5 \pm 9.3$ & 8.937 & $<0.001^{*}$ \\
\hline Access to criteria & $32(71.1 \%)$ & 32 (91.4\%) & 5.079 & $0.024 *$ \\
\hline Highest learning point & $14.2 \pm 1.3$ & $14.8 \pm 0.5$ & 35.925 & $0.009 *$ \\
\hline Long-term recall scores & $12.02 \pm 1.8$ & $13.4 \pm 1.1$ & 6.829 & $<0.001^{*}$ \\
\hline \multicolumn{5}{|l|}{ ERT } \\
\hline Correct disgust (\%) & $27.2 \pm 12.8$ & $26.2 \pm 8.8$ & 4.052 & 0.699 \\
\hline Correct sadness (\%) & $34.6 \pm 12.6$ & $35.8 \pm 14.1$ & 0.589 & 0.699 \\
\hline Correct fear $(\%)$ & $22.7 \pm 8.6$ & $26.8 \pm 7.3$ & 5.530 & 0.253 \\
\hline Correct anger (\%) & $22.7 \pm 8.6$ & $21.6 \pm 4.3$ & 4.238 & 0.511 \\
\hline Correct surprise (\%) & $32.06 \pm 11.4$ & $327 \pm 7.4$ & 4.084 & 0.771 \\
\hline Correct happiness (\%) & $33.8 \pm 10.8$ & $36.7 \pm 10.3$ & 0.023 & 0.229 \\
\hline Total correct (\%) & $62.5 \pm 11.8$ & $67.5 \pm 6.9$ & 5.726 & $0.030 *$ \\
\hline \multicolumn{5}{|l|}{ SST } \\
\hline Proportion of successful stops (\%) & $0.5 \pm 0.1$ & $0.5 \pm 0.1$ & 0.005 & 0.478 \\
\hline \multicolumn{5}{|l|}{ CGT } \\
\hline Deliberation time & $2,670.6 \pm 1,135.2$ & $2,600.7 \pm 966.5$ & 0.323 & 0.772 \\
\hline Quality of decision-making & $0.7 \pm 0.2$ & $0.8 \pm 0.1$ & 2.292 & 0.190 \\
\hline Bet proportion - ascending & $0.4 \pm 2.3$ & $0.4 \pm 0.2$ & 0.365 & 0.775 \\
\hline Bet proportion - descending & $0.6 \pm 0.1$ & $0.6 \pm 0.2$ & 2.926 & 0.116 \\
\hline Overall bet proportion & $0.5 \pm 0.1$ & $0.5 \pm 0.1$ & 0.228 & 0.601 \\
\hline Bet proportion at $6: 4$ & $0.5 \pm 0.2$ & $0.5 \pm 0.2$ & 0.187 & 0.912 \\
\hline Bet proportion at $9: 1$ & $0.6 \pm 0.2$ & $0.6 \pm 0.2$ & 0.094 & 0.409 \\
\hline
\end{tabular}

BPD = borderline personality disorder; CGT = Cambridge Gambling Task; ERT = Emotional Recognition Task; IED = Intra-Extra Dimensional Set Shifting Test; PAL = Paired-Associate Learning Test; RVP = Rapid Visual Processing; SST = Stop-Signal Task; VMPT = Verbal Memory Processes Test.

$* p<0.05$. 
a model consisting of DES-T, motor impulsivity, and SSB scores could significantly represent BPI scores (adjusted $\mathrm{Rs}=0.519, \mathrm{~F}=16.815, \mathrm{p} \leqslant 0.001)$.

The stepwise multivariate regression analysis results for the effects of dissociative experiences are shown in Table 4, the results for the effects of motor impulsivity are shown in Table 5, and the results for the effects of suicidal behavior are shown in Table 6 .

\section{Discussion}

The DSM-5 emphasizes the validity problem in the BPD diagnosis process and suggests the use of hybrid models (including dimensional structures) to resolve it. Our study thus focused on whether the dissociation, impulsivity, and suicidality dimensions were foregrounded in the clinical manifestation of the disorder and whether they were sufficient to explain it. However, we aimed to evaluate neurocognitive functions present in the disorder (including hot cognition, which we believe to better represent social relations and significantly affects the deterioration of interpersonal relationships in BDP) to allow a different approach than previous categorical assessments, as well as to contribute to diagnosis validity (available in the DSM-5).
We detected that dissociation, impulsivity, and suicidality varied from normality in BPD and that these dimensions could significantly explain the clinical manifestation of BPD. Although no model that included these dimensions together could be found in the literature, there was independent discussion of impulsivity, ${ }^{15}$ dissociation ${ }^{16-19}$ and suicidal behavior $^{20}$ playing important roles in BPD.

\section{Comparison of neurocognitive functions in the patient and control groups}

In harmony with other studies, our BPD group showed a disturbance in sustained attention and all parts of verbal memory except for immediate memory, but there was no variation in visual memory/learning performance. A metaanalysis found that though both memory types showed deterioration, visual memory was affected more than verbal memory. ${ }^{6}$ Despite the differing cognitive flexibility results in BPD patients, it is accepted that they frequently experience disturbances. We detected no variation between the BPD and healthy control groups in terms of cognitive flexibility skills. A study using the Intra/Extra Dimensional Set Shifting Test found no variations in BPD. $^{21}$ In a facial emotion recognition study, it was

Table 3 The effects of a model featuring important clinical manifestations of borderline personality disorder

\begin{tabular}{lccccc}
\hline & \multicolumn{5}{c}{ Borderline Personality Inventory Scores } \\
\cline { 2 - 6 } Independent variables & $\beta$ coefficient & $95 \% \mathrm{Cl}$ for $\beta$ coefficient & SE & $t$ & $0.001^{*}$ \\
\hline DES-T & 0.190 & $0.080-0.30$ & 0.054 & 3.488 & $0.028^{*}$ \\
Motor impulsivity & 0.792 & $0.090-1.49$ & 0.348 & 2.278 & $0.038^{*}$ \\
SSB & 0.636 & $0.036-1.24$ & 0.297 & 2.141 & 2.332 \\
Constant & 8.398 & $1.125-15.670$ & 3.601 & $0.025^{*}$ \\
\hline
\end{tabular}

DES-T = Dissociative Experiences Scale - Taxon; SE = standard error; SSB = Scale for Suicidal Behavior.

$* p<0.05$; stepwise multivariate regression analyses were performed.

Table 4 Investigation of the effect of model for clinical features and cognitive functions related to pathological dissociative experiences

\begin{tabular}{|c|c|c|c|c|c|}
\hline \multirow[b]{2}{*}{ Independent variables } & \multicolumn{5}{|c|}{ DES-T } \\
\hline & $\beta$ coefficient & $95 \% \mathrm{Cl}$ for $\beta$ coefficient & SE & $t$ & $p$-value \\
\hline Motor Impulsivity & 3.911 & $2.383-5.438$ & 0.756 & 5.175 & $<0.001^{*}$ \\
\hline ERT (correct sadness) & 0.409 & $0.035-0.784$ & 0.185 & 2.211 & $0.033^{*}$ \\
\hline CGT (decision-making quality) & 24.607 & $1.007-48.207$ & 11.677 & 2.107 & $0.041 *$ \\
\hline IED (PreED errors) & 0.382 & $-0.344-1.108$ & 0.359 & 1.064 & 0.294 \\
\hline Constant & -50.590 & $-81.694-19.486$ & 15.390 & -3.287 & $0.02^{*}$ \\
\hline
\end{tabular}

CGT = Cambridge Gambling Task; DES-T = Dissociative Experiences Scale - Taxon; ERT = Emotion Recognition Task; IED = Intra-Extra Dimensional Set Shifting Test; SE = standard error.

$* p<0.05$; stepwise multivariate regression analyses were performed; adjusted $\mathrm{Rs}=0.420, \mathrm{~F}=8.950, \mathrm{p} \leqslant 0.001$.

Table 5 Investigation of the effect of model for clinical features and cognitive functions related to motor impulsivity

\begin{tabular}{|c|c|c|c|c|c|}
\hline \multirow[b]{2}{*}{ Independent variables } & \multicolumn{5}{|c|}{ Motor impulsivity } \\
\hline & $\beta$ coefficient & $95 \% \mathrm{Cl}$ for $\beta$ coefficient & SE & $t$ & $p$-value \\
\hline SST (proportion of successful stops) & 11.390 & $4.710-18.071$ & 3.310 & 3.441 & $0.001^{*}$ \\
\hline ERT (total correct) & -0.072 & $-0.144-0.00$ & 0.035 & -2.031 & $0.049 *$ \\
\hline Constant & 10.340 & $4.722-15.957$ & 2.784 & 3.714 & $0.001 *$ \\
\hline
\end{tabular}

ERT = Emotional Recognition Task; SE = standard error; SST = Stop-Signal Task.

$* p<0.05$; stepwise multivariate regression analyses were performed; Adjusted $R s=0.233, F=7.674, p=0.001$. 
Table 6 Investigation of the effect model for clinical features and cognitive functions related to suicidal behavior

\begin{tabular}{|c|c|c|c|c|c|}
\hline \multirow[b]{2}{*}{ Independent variables } & \multicolumn{5}{|c|}{ SSB scores } \\
\hline & $\beta$ coefficient & $95 \% \mathrm{Cl}$ for $\beta$ coefficient & SE & $t$ & $p$-value \\
\hline CGT bet proportion - descending & 10.484 & $5.730-15.238$ & 2.298 & 4.562 & $\leqslant 0.001 *$ \\
\hline CGT bet proportion $\% 50$ probability & -5.628 & $-9.252-2.004$ & 1.752 & -3.213 & $0.004^{*}$ \\
\hline VMPT (immediate memory) & 0.657 & $0.195-1.119$ & 0.223 & 2.944 & $0.007 *$ \\
\hline Constant & -2.874 & $-7.026-1.278$ & 2.007 & -1.432 & 0.166 \\
\hline
\end{tabular}

CGT = Cambridge Gambling Test; SE = standard error; SSB = Scale for Suicidal Behavior; VMPT = Verbal Memory Processes Test.

$* p<0.05$; stepwise multivariate regression analyses were performed; Adjusted Rs $=0.506, F=9.893 p \leqslant 0.001$.

observed that emotion recognition skills were generally decreased in BPD. ${ }^{22}$ We found that separate recognition of the six basic facial expressions did not significantly vary in BPD, although patient performance for total emotion recognition was lower. The lowa Gambling Task, which assesses decision implementation and impulsivity processes on a cognitive level and in a manner integrated with emotion, has frequently been used in studies assessing decision implementation skills. ${ }^{23}$ In the present study, we used the CGT, which is reported to be less affected by working memory and learning status than the lowa Gambling Task. We detected no variation in decisionmaking skills between the BPD and control groups. In our study, there was no significant difference in Stop-Signal Task scores between the BPD and control groups. The results of other studies involving the Stop-Signal Task harmonize with ours. ${ }^{24,25}$

The BPD group had lower sustained attention, facial emotion recognition, and verbal memory functions in their neurocognitive profile than healthy controls. No variation was observed in cognitive flexibility, visual learning/memory, response inhibition, or decision-making skills between the groups.

\section{Neurocognitive profile in the dissociation dimension}

In cases of intense stress exposure, temporary dissociative symptoms can be seen in patients with borderline personality disorder. ${ }^{26}$ It has been reported that the dissociative symptoms in BPD are related to variation in the parietal cortex ${ }^{27}$ and are mostly caused by difficulties in autobiographical memory recall. ${ }^{28}$ In another study, no relation was observed between dissociation scores, visual perception, and working memory. ${ }^{29}$ We believe that BPD patients are a heterogeneous group and that the nonstandardized test material has led to conflicting research results. Our study found that motor impulsivity, decision implementation (choosing the appropriate response) and emotion recognition significantly predicted the pathological indicators observed in BPD. Haaland et al. ${ }^{30} \mathrm{com}$ pared healthy controls and BPD patients with and without dissociative symptoms, including an examination of cognitive areas such as attention, working memory, verbal memory, visual memory, and executive function. The authors reported that BPD patients with dissociative symptoms performed worse than healthy subjects in all cognitive domains. They emphasized that patients with dissociative symptoms performed worse than those without dissociative symptoms in executive function, working memory, and verbal memory areas, concluding that pathological dissociation could be a clinical variable that differentiates BPD patients in terms of cognitive functioning. These authors found a negative relationship between DES-T scores, attention, and verbal memory, and they found no relationship between decision implementation, cognitive flexibility, and visual memory. ${ }^{30}$ Although they found that both cognitive areas were associated with dissociative symptoms and their comparison with healthy individuals resulted in different features than the current study, it should be pointed out that their sample size was very small. ${ }^{30}$ Thus, these findings may not reach significance in comparison and correlation tests.

\section{Neurocognitive profile in the impulsivity dimension}

It was observed that the basal metabolic rate of the orbitofrontal cortex area decreased in BPD. ${ }^{9}$ The orbitofrontal cortex is involved in executive functions that regulate decision implementation ${ }^{31}$ and impulsive behaviors. Functioning along with the orbitofrontal cortex in decision implementation, ${ }^{32}$ the amygdala also enables correct recognition of emotions. ${ }^{33}$ Impulsivity is a behavioral characteristic of BPD.

We detected that response inhibition and facial emotion recognition might significantly predict impulsivity. The association between impulsivity and response inhibition has been examined in other psychiatric disorders, and it was concluded that impulsivity and response inhibition probably occurred through the same biological mechanisms in the prefrontal cortex. ${ }^{34}$ A study on the relationship between impulsivity and facial emotion recognition in ADHD and ASD patients emphasized that ADHD symptoms had an effect on emotion recognition. ${ }^{35}$ The same study also reported that impulsivity and emotion recognition had an inverse interaction. Although research on this topic is very limited, our findings support those of previous studies.

\section{Neurocognitive profile in the suicidality dimension}

Suicide threats and attempts are an important diagnostic criterion in BPD. ${ }^{36}$ BPD has a high mortality due to suicide. In the present study, cognitive functions, such as decision-making and immediate memory, were found to have an effect on suicidality. In a study by Bazanis et al., ${ }^{37} 42$ BPD patients with self-injurious behavior were compared with healthy controls, and the only differences found between the two groups were disruptions in decision making and planning. BPD patients took longer to decide, selected the most unlikely outcomes, and placed 
earlier bets on whether their choices were correct, demonstrating disinhibited responses. The authors argued that these deficits may be caused by disturbances in the orbitofrontal and dorsolateral frontal brain regions, and that a general aversion to delay may be an important feature of BPD. ${ }^{37}$ Therefore, assessing decision-making impairment and verbal memory deficits may be important in predicting and preventing current suicidal behavior in BPD patients. Nevertheless, to determine whether the model predicts future suicidal behavior, prospective studies including patients with the specified neurocognitive profile are needed.

However, it should be noted that the present findings may not be generalizable. LeGris et al. stated that suicidal behavior was related only to the Stroop test among neurocognitive functions. ${ }^{7}$ On the other hand, Ghanem et al. found that suicidal behavior was not related to cognitive flexibility, which is an executive function. ${ }^{5}$ The fact that our sample consisted only of patients from our hospital's psychotherapy center may be regarded a limitation. Almost half of the patients $(46.7 \%)$ had received at least 6 months of psychotherapy or regular polyclinic follow-up. Due to the fact that these patients had a positive course, such as remaining on the waiting list, the sample may not be representative of all BPD patients. Furthermore, due to our small sample size, our findings require confirmation by further studies. Although patients with low intelligence or cognitive deficits were excluded through a psychiatric interview, the lack of a detailed IQ examination should be considered a limitation. Although our study aimed to provide an integrated assessment of the disorder, the relatively long tests we used may have been challenging for the patients to finish, despite motivation-boosting interventions and breaks between the neurocognitive tests.

In conclusion, we found that the dissociation, impulsivity, and suicidality dimensions are sufficient to represent BPD manifestation. It was demonstrated that these dimensions vary in neurocognitive profiles and interact with clinical dimensions. Due to the heterogenic manifestation of psychiatric disorders, cognitive functions are hard to assess. Accordingly, it is believed that separate assessments of the symptoms and examination of their interaction with cognitive functions may yield more consistent data. The results of this study support the idea that different dimensions of BPD carry different neurocognitive profiles and require examination from a dimensional perspective. Longitudinal studies on neurocognitive functions involving dimensional approach are needed to confirm the diagnostic validity of these results.

\section{Disclosure}

The authors report no conflicts of interest.

\section{References}

1 Skodol AE, Siever LJ, Livesley WJ, Gunderson JG, Pfohl B, Widiger TA. The borderline diagnosis II: biology, genetics and clinical course. Biol Psychiatry. 2002;51:951-63.
2 Stein DJ, Hollander E, Liebowitz MR. Neurobiology of impulsivity and the impulse control disorders. J Neuropsychiatry Clin Neurosci. 1993;5: 9-17.

3 Paris J. The diagnosis of borderline personality disorder: problematic but better than the alternatives. Ann Clin Psychiatry. 2005;17: 41-6.

4 Paris J. Personality disorders over time: precursors, course, and outcome. J Pers Disord. 2003;17:479-88.

5 Ghanem M, El-Serafi D, Sabry W, El Rasheed AH, Razek GA, Soliman A, et al. Executive dysfunctions in borderline personality disorder: correlation with suicidality and impulsivity. Middle East Curr Psychiatry. 2016;23:85-92.

6 Ruocco AC. The neuropsychology of borderline personality disorder: a meta-analysis and review. Psychiatry Res. 2005;137:191-202.

7 LeGris J, van Reekum R. The neuropsychological correlates of borderline personality disorder and suicidal behaviour. Can J Psychiatry. 2006;51:131-42.

8 Kunert HJ, Druecke HW, Sass H, Herpertz SC. Frontal lobe dysfunctions in borderline personality disorder? Neuropsychological findings. J Pers Disord. 2003;17:497-509.

9 Soloff PH, Meltzer CC, Becker C, Greer PJ, Kelly TM, Constantine D. Impulsivity and prefrontal hypometabolism in borderline personality disorder. Psychiatry Res. 2003;123:153-63.

10 Korzekwa MI, Dell PF, Links PS, Thabane L, Fougere P. Dissociation in borderline personality disorder: a detailed look. J Trauma Dissociation. 2009;10:346-67.

11 Mak AD, Lam LC. Neurocognitive profiles of people with borderline personality disorder. Curr Opin Psychiatry. 2013;26:90-6.

12 Domes G, Schulze L, Herpertz SC. Emotion recognition in borderline personality disorder - a review of the literature. J Pers Disord. 2009;23: 6-19.

13 Faul F, Erdfelder E, Lang AG, Buchner A. G*Power 3: a flexible statistical power analysis program for the social, behavioral, and biomedical sciences. Behav Res Methods. 2007;39:175-91.

14 Faul F, Erdfelder E, Buchner A, Lang AG. Statistical power analyses using $G^{\star}$ Power 3.1: tests for correlation and regression analyses. Behav Res Methods. 2009;41:1149-60.

15 Hochhausen NM, Lorenz AR, Newman JP. Specifying the impulsivity of female inmates with borderline personality disorder. J Abnorm Psychol. 2002;111:495-501.

16 Zlotnick C, Johnson DM, Yen S, Battle CL, Sanislow CA, Skodol AE, et al. Clinical features and impairment in women with borderline personality disorder (BPD) with posttraumatic stress disorder (PTSD), BPD without PTSD, and other personality disorders with PTSD. J Nerv Ment Dis. 2003;191:706-13.

17 Zanarini MC, Ruser TF, Frankenburg FR, Hennen J, Gunderson JG. Risk factors associated with the dissociative experiences of borderline patients. J Nerv Ment Dis. 2000;188:26-30.

18 Simeon D, Nelson D, Elias R, Greenberg J, Hollander E. Relationship of personality to dissociation and childhood trauma in borderline personality disorder. CNS Spectr. 2003;8:755-62.

19 Shearer SL. Dissociative phenomena in women with borderline personality disorder. Am J Psychiatry. 1994;151:1324-8.

20 Black DW, Blum N, Pfohl B, Hale N. Suicidal behavior in borderline personality disorder: prevalence, risk factors, prediction, and prevention. J Pers Disord. 2004;18:226-39.

21 Gvirts HZ, Braw Y, Harari H, Lozin M, Bloch Y, Fefer K, et al. Executive dysfunction in bipolar disorder and borderline personality disorder. Eur Psychiatry. 2015;30:959-64.

22 Daros AR, Zakzanis KK, Ruocco AC. Facial emotion recognition in borderline personality disorder. Psychol Med. 2013;43:1953-63.

23 Bechara A, Damasio AR, Damasio H, Anderson SW. Insensitivity to future consequences following damage to human prefrontal cortex. Cognition. 1994;50:7-15.

24 Legris J, Links PS, van Reekum R, Tannock R, Toplak M. Executive function and suicidal risk in women with borderline personality disorder. Psychiatry Res. 2012;196:101-8.

25 Thomsen MS, Ruocco AC, Carcone D, Mathiesen BB, Simonsen E. Neurocognitive deficits in borderline personality disorder: associations with childhood trauma and dimensions of personality psychopathology. J Pers Disord. 2017;31:503-21.

26 American Psychiatric Association. Diagnostic and Statistical Manual of Mental Disorders, Fourth Edition, Text Revision (DSM-IV-TR). Arlington: American Psychiatric Publishing; 2000. 
27 Irle E, Lange C, Sachsse U. Reduced size and abnormal asymmetry of parietal cortex in women with borderline personality disorder. Biol Psychiatry. 2005;57:173-82.

28 Jones B, Heard H, Startup M, Swales M, Williams JM, Jones RS. Autobiographical memory and dissociation in borderline personality disorder. Psychol Med. 1999;29:1397-404.

29 Stevens A, Burkhardt M, Hautzinger M, Schwarz J, Unckel C. Borderline personality disorder: impaired visual perception and working memory. Psychiatry Res. 2004;125:257-67.

30 Haaland VO, Landro NI. Pathological dissociation and neuropsychological functioning in borderline personality disorder. Acta Psychiatr Scand. 2009;119:383-92.

31 Bechara A, Damasio H, Damasio AR. Emotion, decision making and the orbitofrontal cortex. Cereb Cortex. 2000;10:295-307.

32 van Elst LT, Hesslinger B, Thiel T, Geiger E, Haegele K, Lemieux L, et al. Frontolimbic brain abnormalities in patients with borderline personality disorder: a volumetric magnetic resonance imaging study. Biol Psychiatry. 2003;54:163-71.
33 Adolphs R, Spezio M. Role of amygdala in processing visual social stimuli. Prog Brain Res. 2006;156:363-78.

34 Christodoulou T, Lewis M, Ploubidis GB, Frangou S. The relationship of impulsivity to response inhibition and decision-making in remitted patients with bipolar disorder. Eur Psychiatry. 2006;21: 270-3.

35 Sinzig J, Morsch D, Lehmkuhl G. Do hyperactivity, impulsivity and inattention have an impact on the ability of facial affect recognition in children with autism and ADHD? Eur Child Adolesc Psychiatry. 2008;17:63-72.

36 American Psychiatric Association Practice Guidelines. Practice guideline for the treatment of patients with borderline personality disorder. American Psychiatric Association. Am J Psychiatry. 2001; 158(10 Suppl):1-52.

37 Bazanis E, Rogers RD, Dowson JH, Taylor P, Meux C, Staley C, et al. Neurocognitive deficits in decision-making and planning of patients with DSM-III-R borderline personality disorder. Psychol Med. 2002;32:1395-405. 